\title{
Information is not about measurability
}

\author{
Juan Dubra ${ }^{\mathrm{a}}$, Federico Echenique ${ }^{\mathrm{b}, *}$ \\ ${ }^{a}$ Universidad de Montevideo, Prudencio de Pena 2440, Montevideo CP11600, Uruguay \\ ${ }^{\mathrm{b}}$ Division of Humanities and Social Sciences, MC 228-77, California Institute of Technology, Pasadena, \\ CA 91125, USA
}

Received 1 October 2002; received in revised form 1 May 2003; accepted 1 June 2003

\begin{abstract}
We present a simple example where the use of $\sigma$-algebras as a model of information leads to a paradoxical conclusion: a decisionmaker prefers less information to more. We then explain that the problem arises because the use of $\sigma$-algebras as the informational content of a signal is inadequate. We provide a characterization of the different models of information in the literature in terms of Blackwell's theorem.

(C) 2003 Elsevier B.V. All rights reserved.
\end{abstract}

Keywords: Blackwell's theorem; Measurability; Models of information; Partitions; Information-preserving; $\sigma$-algebras

JEL classification: $\mathrm{C} 60 ; \mathrm{C} 70 ; \mathrm{G} 12$

\section{Introduction}

Algebras of events are closed under finite unions; $\sigma$-algebras are closed under countable unions. It is well-known that algebras are not rich enough for most purposes in probability theory. It is slightly less known — but trivial - that they are not rich enough to model an agents' information.

Researchers in statistics, finance and economics use $\sigma$-algebras to model an agents' information because $\sigma$-algebras allow the use of probability-theory tools. We point out that, as a model of information, $\sigma$-algebras are just as problematic as algebras.

\footnotetext{
*Corresponding author. Tel.: +1-626-395-4273; fax: +1-626-405-9841.

E-mail addresses: dubraj@um.edu.uy (J. Dubra), fede@hss.caltech.edu (F. Echenique).
} 
We present a simple example where the use of $\sigma$-algebras as a model of information leads to a paradoxical conclusion: a decisionmaker prefers less information to more. We then explain why the problem arises, and provide a characterization of the different models of information in the literature in terms of Blackwell's theorem.

\subsection{The example}

Let the state of the world be a real number between 0 and 1 , so the set of possible states is $\Omega=[0,1]$. Suppose that a decisionmaker can choose to either be perfectly informed, so that she gets to know the exact value of $\omega$, or only be told if the true $\omega$ is smaller or larger than $1 / 2$. In the first case, the information can be modeled as the partition of all elements of $\Omega, \tau=\{\{\omega\}: \omega \in \Omega\}$. In the second case, the information is the partition $\tau^{\prime}=\{[0,1 / 2)$; $[1 / 2,1]\}$.

(a) Suppose we were to model the informational content of $\tau$ and $\tau^{\prime}$ by the algebras generated by $\tau$ and $\tau^{\prime}$; denote these by $A(\tau)$ and $A\left(\tau^{\prime}\right)$. It is easy to see that $A\left(\tau^{\prime}\right)=\{\emptyset$; $\Omega ;[0,1 / 2) ;[1 / 2,1]\}$, while $A(\tau)$ is the collection of sets in $[0,1]$ that are either finite or have finite complement.

The first problem with algebras as a model of information is that $A(\tau)$ is not finer than $A\left(\tau^{\prime}\right)$ : a decisionmaker with information $A\left(\tau^{\prime}\right)$ may get to learn that the event $[0,1 / 2)$ has happened; a decisionmaker with information $A(\tau)$ never learns if $[0,1 / 2)$ has happened or not because $[0,1 / 2) \notin A(\tau)$. The second - and more important - problem with algebras as a model of information is that it can predict the wrong choice by a decisionmaker.

Suppose the decisionmaker is a risk-neutral expected-utility maximizer. The individual must first choose between $\tau$ and $\tau^{\prime}$, and then decide to buy either a bond or a stock. The return on the stock is $S(\omega)=\omega$. The bond yields $3 / 8$ in every state of the world. Suppose in addition that the state of the world is chosen according to a uniform distribution on $[0,1]$.

The decisionmaker evaluates $S$ according to $E\left(S \mid A(\tau)\right.$ ) (or $E\left(S \mid A\left(\tau^{\prime}\right)\right)$ ), as the conditional expectation is the decisionmaker's prediction based on the informational content of $\tau$ or $\tau^{\prime}$. Since $E(S \mid A(\tau))$, viewed as a random variable, must be measurable with respect to $A(\tau)$ — which consists of events with probability 0 or 1 (i.e. essentially of $\{\emptyset, \Omega\}$ - -it must be a.s. constant and equal to $1 / 2$. See Appendix A for a proof. It is immediate that

$$
E\left(S \mid A\left(\tau^{\prime}\right)\right)(\omega)= \begin{cases}1 / 4 & \text { if } \omega<1 / 2 \\ 3 / 4 & \text { otherwise }\end{cases}
$$

In almost every state of the world $E(S \mid A(\tau))=1 / 2$ is larger than the return to the bond $(3 / 8)$, so if the individual decides to observe information $\tau$ she will a.s. buy the stock. The expected utility of choosing $\tau$ is thus $1 / 2$. On the other hand if she chooses $\tau^{\prime}$, she will buy the bond when $\omega<1 / 2$ and the stock if $\omega \geq 1 / 2$, and will thus get a utility of $(3 / 8)(1 / 2)+(3 / 4)(1 / 2)=9 / 16>1 / 2$.

Then, while $\tau$ is obviously more informative than $\tau^{\prime}$, the decisionmaker strictly prefers $\tau^{\prime}$ over $\tau$. The reason is that the algebra generated by $\tau$, and used in forming $E(S \mid A(\tau))$, is not informative at all: it is a collection of trivial sets, having either 
probability 0 or 1 . The algebra generated by $\tau^{\prime}$, on the other hand, distinguishes $[0,1 / 2)$ from $[1 / 2,1]$.

(b) The problem discussed in (a) does not disappear if we use $\sigma$-algebras instead of algebras: suppose that the decisionmaker evaluates $S$ according to $E(S \mid \sigma(\tau))$ or $E(S \mid \sigma$ $\left(\tau^{\prime}\right)$ ), where $\sigma(\tau)$ is the $\sigma$-algebra generated by $\tau$. ${ }^{1}$ It is still true (see Appendix A) that $E(S \mid \sigma(\tau))=1 / 2$ a.s., while $E\left(S \mid \sigma\left(\tau^{\prime}\right)\right)$ is $1 / 4$ on $[0,1 / 2)$ and $3 / 4$ on $[1 / 2,1]$. If the individual decides to observe information $\tau$ she will a.s. buy the stock - the expected utility of choosing $\tau$ is $1 / 2$, and the decisionmaker still prefers $\tau^{\prime}$ over $\tau$.

The problem now is that $\sigma(\tau)$ is still trivial; it is the collection of sets that are either countable or have countable complement.

\subsection{Our point}

The problem in our example is that finer partitions need not generate finer algebras or $\sigma$-algebras. We show below that algebras and $\sigma$-algebras do not preserve information because they are not closed under arbitrary unions. Heuristically, if the decisionmaker 'knows' that some collection $\mathscr{E}$ of events is false, she should 'know' that their union is false. If $\mathscr{E}$ is infinite, however, the union of events in $\mathscr{E}$ need not be in the algebra, and if $\mathscr{E}$ is uncountable, the union of events in $\mathscr{E}$ need not be in the $\sigma$-algebra.

As is well-known, there are many technical problems with 'large' $\sigma$-algebras - and closedness under arbitrary unions would generally deliver large $\sigma$-algebras. We have no solution to offer, but we think it is important to document and explain these problems with the interpretation of $\sigma$-algebras.

We do not argue that using $\sigma$-algebras as the informational content of signals is always inappropriate. We only want to emphasize that one should be careful when using $\sigma$-algebras as the informational content of signals. We believe that this is relevant, as even technically able economists ignore the problems we emphasize (see the discussion in Section 3).

\subsection{Notation and definitions}

A partition $\tau$ of a set $\Omega$ is a collection of pairwise disjoint subsets whose union is $\Omega$; note that for each state of nature $\omega$ there is a unique element of $\tau$ that contains $\omega$. A decisionmaker whose information is represented by $\tau$ is informed only that the element of $\tau$ that contains the true state of nature has occurred. In other words, the decisionmaker cannot distinguish between states that belong to the same element of $\tau$. If $\tau, \tau^{\prime}$ are partitions, say that $\tau^{\prime}$ is finer than $\tau$, written $\tau^{\prime} \geq \tau$, if for every $C \in \tau^{\prime}$ there is $B$ in $\tau$ such that $C \subseteq B$. For any collection $\mathscr{C}$ of subsets of $\Omega$, the $\sigma$-algebra generated by $\mathscr{C}$, denoted $\sigma(\mathscr{C})$, is the smallest $\sigma$-algebra that contains $\mathscr{C}$.

We can define information structures to be signals $f: \Omega \rightarrow Y$, for some set $Y$ of observable signal values. This way of modeling information is equivalent to using

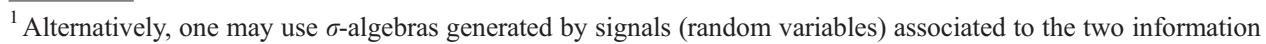
structures. As we discuss in Section 3.1, the same phenomenon shows up. See our comment in Section 3.4 for a third interpretation of the informational content of $\sigma$-algebras.
} 
partitions: each signal generates a partition, and each partition can be interpreted as a signal. Define the partition $P_{f}$ of $\Omega$ associated to $f$ by

$$
P_{f}=\left\{f^{-1}(y): y \in Y\right\} .
$$

A pair $(\Omega, \mathscr{F})$, where $\mathscr{F}$ is a $\sigma$-algebra of subsets of $\Omega$, is a measurable space. Let $(\Omega, \mathscr{F})$ and $(Y, \mathscr{G})$ be two measurable spaces. A function $f: \Omega \rightarrow Y$ is measurable if $f^{-1}(B) \in \mathscr{F}$ for all $B \in \mathscr{G}$. The $\sigma$-algebra generated by $f$, denoted $\sigma(f, \mathscr{G})$, is the smallest $\sigma$-algebra on $\Omega$ for which $f$ is measurable. We say that a $\sigma$-algebra $\mathscr{B}$ on $Y$ distinguishes $f$ if for all $\omega \in \Omega, f(\omega) \in \mathscr{B}$.

For a given set of states of nature $\Omega$ and an arbitrary measurable set $(Y, \mathscr{B})$, a collection $\alpha=\left(m_{\omega}\right)_{\omega \in \Omega}$ of probability measures on $(Y, \mathscr{B})$ is an experiment. If $\mathscr{B}, \mathscr{C}$ are $\sigma$-algebras on $Y, W$, respectively, a stochastic transformation $T$ is a function $Q(y, E)$ defined for all $y \in Y$ and $E \in \mathscr{C}$ which for fixed $E$ is a measurable function of $y$ and for fixed $y$ is a probability measure on $\mathscr{C}$. For any probability measure $m$ on $\mathscr{B}$, the function

$$
M(E)=\int Q(y, E) \mathrm{d} m(y)
$$

is a probability measure on $\mathscr{C}$, denoted by $\operatorname{Tm}$. If $\alpha=\left(m_{\omega}\right)_{\omega \in \Omega}$ and $\beta=\left(M_{\omega}\right)_{\omega \in \Omega}$ are two experiments, with $m_{\omega}, M_{\omega}$ defined on $\mathscr{B}$ and $\mathscr{C}$, respectively, we shall say that $\alpha$ is sufficient for $\beta$, or $\beta$ is a garbling of $\alpha$, written $\alpha \succ \beta$ if there exists a stochastic transformation $T$ such that $\operatorname{Tm}_{\omega}=M_{\omega}$ for all $\omega$. For $f: \Omega \rightarrow Y_{f}$ and $g: \Omega \rightarrow Y_{g}$, we will say that $g$ is a garbling of $f$ if $\beta=\left(\delta_{g(\omega)}\right)$ is a garbling of $\alpha=\left(\delta_{f(\omega)}\right)$. To understand this definition, notice that an experiment is just a function from $\Omega$ to the set of probability measures on some space $\left(Y_{f}, \mathscr{B}\right)$. Then, a signal $f: \Omega \rightarrow Y_{f}$ can be identified with the experiment that associates with each $\omega$, the lottery which is degenerate in $f(\omega)$.

\section{A Blackwell theorem}

Consider the following set-up.

- $\Omega$ is the set of states of nature;

- $Z$ is the set of consequences, $Z$ has at least two elements;

- an act is a function $a: \Omega \rightarrow Z, A=Z^{\Omega}$ is the set of all acts;

- a decisionmaker is a preference relation $\succeq$ on $A$ ( $\succeq$ is a complete transitive binary relation on $A$ ).

The information structures available to a decisionmaker are signals $f: \Omega \rightarrow Y_{f}$ for some space $Y_{f}$. The decisionmaker is informed of the value taken by $f$ and she must then choose a consequence in $Z$. An act $a: \Omega \rightarrow Z$ is $f$-feasible if $a(\omega)=a\left(\omega^{\prime}\right)$ whenever $f(\omega)=f\left(\omega^{\prime}\right)$.

A decisionmaker $\succeq$ prefers signal $f$ to $g$ if and only if, for any $g$-feasible act $a$, there exists an $f$-feasible act $\tilde{a}$ such that $\tilde{a} \succeq a$. 
Theorem A. Let $f: \Omega \rightarrow Y_{f}$ and $g: \Omega \rightarrow Y_{g}$. The following are equivalent.

1. Every decisionmaker prefers $f$ to $g$;

2. $P_{f}$ is finer than $P_{g}$;

3. there is $h: Y_{f} \rightarrow Y_{g}$ such that $g=h \circ f$;

4. $g$ is a garbling of $f$;

5. the $\sigma$-algebra of arbitrary unions of elements in $P_{f}$ is finer than the $\sigma$-algebra of arbitrary unions of elements in $P_{g}$;

6. for all $\sigma$-algebras $\mathscr{B}, \mathscr{C}$ on $Y_{f}, Y_{g}$ that distinguish $f$ and $g$, and are closed under arbitrary unions, $\sigma(f, \mathscr{B})$ is finer than $\sigma(g, \mathscr{C})$.

Remark 1. Blackwell's (1951) theorem is the equivalence of 1 and 4, in the context of 'noisy signals': his experiments are functions from $\Omega$ to the set of probability measures on some space $X$. By enlarging the state-space, his context can be embedded in ours, but the statement in Blackwell (1951) does not follow from our Theorem A applied to the enlarged state-space. On the other hand, Blackwell's theorem does not imply $1 \Leftrightarrow 4$ in the present context, as his theorem was for a finite state space (this is also true of the version in Blackwell, 1953). Therefore, neither theorem is more general.

Remark 2. We believe that the equivalence of 1,2,3, and 4 in Theorem $\mathrm{A}$ is known, but we are unaware of a statement or proof in print. In any case, we are interested in the equivalence of 1 with 5 and 6 as an explanation of our example in 1.1.

Theorem A explains the paradox in our example. Signal $\tau$ is more informative than $\tau^{\prime}$, but the $\sigma$-algebra generated by $\tau$ is not finer than the $\sigma$-algebra generated by $\tau^{\prime}$. The root of the problem is very simple: if the decisionmaker knows that no $\omega$ with $\omega<1 / 2$ has occurred, any model of information should prescribe that the decisionmaker knows that $[0$, $1 / 2$ ) has not occurred. But, if one models the decisionmaker's information as $\sigma(\tau)$, the decisionmaker can never 'know' that the event $[0,1 / 2)$ occurred or not, as $[0,1 / 2) \notin \sigma(\tau)$. Knowledge is closed under arbitrary unions, but $\sigma$-algebras need not be.

\section{Discussion}

1. A common alternative to modeling the information content of a signal $f: \Omega \rightarrow Y_{f}$, when $Y_{f}$ is endowed with a $\sigma$-algebra $C$, is $\sigma(f, \mathscr{C})$, the $\sigma$-algebra generated by $f$. By Theorem A this construction will preserve information if $\sigma$-algebras on target spaces are closed under arbitrary unions. If this is not the case, it is easy to generate examples where $\sigma(f, \mathscr{C})$ does not preserve information. In fact, by choosing the $\sigma$-algebras $\mathscr{C}$ and $\mathscr{B}$ on target spaces appropriately, we can reproduce the example in the Introduction with signals $f, g$, and conditional expectations $E(S \mid \sigma(f, \mathscr{C}))$ and $E(S \mid \sigma(g, \mathscr{B})){ }^{2}$

\footnotetext{
${ }^{2}$ The signals $f, g: \Omega \rightarrow \Omega$, where $f$ is the identity and $g$ is the indicator function of the interval $[0,1 / 2)$ work, when $\Omega$ is endowed with the countable-co-countable $\sigma$-algebra. Signal $f$ is more informative than $g$, but the decisionmaker ends up preferring $g$.
} 
As an illustration, consider the following situation. Suppose $Y_{f}=Y_{g}=[0,1]$, and let $\mathscr{F}$ be any $\sigma$-algebra of subsets of $[0,1]$ such that $\{1\} \in \mathscr{F} \neq 2^{[0,1]}$. If $f: \Omega \rightarrow Y_{f}$ is the identity, and $g=\chi_{E}$ for some $E \in 2^{[0,1]} \backslash \mathscr{F}$, we obtain $\sigma(f, \mathscr{F})=\mathscr{F}$, and $\sigma(g, \mathscr{F})=$ $\left\{\emptyset, E, E^{c},[0,1]\right\}$. Thus $\sigma(f, \mathscr{F})$ and $\sigma(g, \mathscr{F})$ are not comparable, while $f$ is more informative than $g$.

This example shows the same problems as those in the Introduction may arise when $\mathscr{F}$ is chosen appropriately. The results of Blackwell (1956) show that under some regularity conditions on $(\Omega, B)$ and the $\sigma$-algebras on $Y_{f}$ and $Y_{g}$, the $\sigma$-algebra generated by $f$ is finer than that generated by $g$. This raises the issue of what $\sigma$-algebra should be used on the target space. Unfortunately, we do not have a preference-based theory for selecting among alternative $\sigma$-algebras on target spaces. ${ }^{3}$ An additional problem with using $\sigma(f, \mathscr{F})$ as the informational content of $f$, is that $\sigma(f, \mathscr{F})$ changes when $\mathscr{F}$ changes, even though the informational content of $f$ does not.

One could interpret $\mathscr{C}$ as the events that the decisionmaker can observe, and use this interpretation in order to choose among different $\sigma$-algebras on the target spaces. But then $\mathscr{C}$ should also be closed under arbitrary unions, both to preserve information (Theorem A) and because 'perception'-much like 'knowledge'—should be closed under arbitrary unions.

2. When $\Omega$ is countable, Theorem A shows that $\sigma$-algebras preserve information, as any union of sets in $\Omega$ can at most be countable. Many models, though, require an uncountable number of states of nature. This is the case, for example, of Savage's model of decision under uncertainty (if we want subjective probabilities to be countably additive, as is usually the case in economics), or of games of incomplete information (Mertens and Zamir, 1985; Brandenburger and Dekel, 1993). In other models, an uncountable space is necessary to use calculus methods.

3. The information-preserving $\sigma$-algebras of arbitrary unions have two well-known disadvantages (see e.g. Dudley, 1989): they may be too large for some countably additive measures to be well-defined, and they have no clear links to the spaces' topological properties, like the Borel.

4. Stinchcombe (1990) proves that, in the spaces that Blackwell (1956) introduced, a countably generated $\sigma$-algebra $\mathscr{F}$ can be identified with the partition

$$
\{\cap\{B: B \in \mathscr{F}, \omega \in B\}: \omega \in \Omega\}
$$

of its atoms, and that all $\sigma$-algebras are 'close' to a countably generated $\sigma$-algebra. ${ }^{4}$ In this particular sense, then, arbitrary $\sigma$-algebras possess an informational content.

5. Our example has some similarities with Example 4.10 in Billingsley (1995). Billingsley argues that the interpretation of $\sigma$-algebras as information is weak, using the following argument. Firstly, he notices that the countable-co-countable $\sigma$-algebra $\mathscr{F}$ generates the finest partition of $[0,1]$ (two states belong to the same element of the partition if no $B \in \mathscr{F}$ distinguishes between them). In that sense, $\mathscr{F}$ contains 'all the information'. Secondly, he notices that for every $B \in \mathscr{F}$ and any Lebesgue measurable $C$, the

\footnotetext{
${ }^{3}$ We thank Larry Epstein, Peter Fishburn, Itzak Gilboa, Massimo Marinacci and Peter Wakker for their feedback on this issue.

${ }^{4}$ We are grateful to Maxwell Stinchcombe for pointing this out.
} 
Lebesgue probability of $C$ conditional on $B$ is just the Lebesgue probability of $C$. In that sense, $\mathscr{F}$ contains no information at all.

There is one important difference between Billingsley's example and our example: we argue that what makes the connection between information and $\sigma$-algebras weak is that finer partitions need not generate finer $\sigma$-algebras; Billingsley's argument is concerned with the partitions generated by $\sigma$-algebras. This distinction is relevant because different partitions generate different $\sigma$-algebras, but different $\sigma$-algebras may generate the same partition. In particular, Billingsley's example does not imply that Blackwell's theorem is false when information is modeled as $\sigma$-algebras - our example does.

6. The results in this paper are very simple, the question remains if they are not 'known', or part of some oral tradition-we are confident they have not appeared in print. Yannelis (1991) claims that finer partitions generate finer $\sigma$-algebras. We do not wish to claim that any of his results are false, but we do believe Yannelis' paper is proof that our points are original.

\section{Acknowledgements}

We are grateful to an anonymous referee, David Blackwell, Don Brown, Héctor Chade, David Levine, Jim Pitman, Maxwell Stinchcombe, Tarun Sabarwal, Bill Sudderth, Sergio Turner, Bill Zame, and seminar participants at UCLA and Yale for comments. We thank Larry Epstein, Peter Fishburn, Itzak Gilboa, Catherine Huafei Yan, Massimo Marinacci, Ernesto Mordecki, Malempati Rao, Srinivasa Varadhan and Peter Wakker for their kind attention to our questions. Part of this work was done while Juan Dubra visited Yale University, and Federico Echenique visited U.C. Berkeley.

\section{Appendix A}

Proof of $E(S \mid \sigma(\tau))=1 / 2$ in the example of the Introduction. We shall show that any version of $E(S \mid \sigma(\tau))$ is a.s. equal to $1 / 2$. Let $j: \Omega \rightarrow \mathrm{R}$ be any version of $E(S \mid \sigma(\tau))$. We shall show that $j^{-1}(1 / 2,+\infty)$ and $j^{-1}(-\infty, 1 / 2)$ are countable sets; this suffices as countable sets have probability zero. We first show that, for arbitrary natural $n, j^{-1}$ $[1 / 2+1 / n,+\infty)$ is countable. Suppose it is uncountable, then $\left(j^{-1}[1 / 2+1 / n,+\infty)\right)^{c}$ is countable, because $j$ is $\sigma(\tau)$ - measurable and $\sigma(\tau)$ is the countable-co-countable $\sigma$ algebra. Then $j^{-1}[1 / 2+1 / n,+\infty)$ has measure one and we obtain,

$$
\frac{1}{2}=\int_{j^{-1}[1 / 2+1 / n,+\infty)} j(\omega) \mathrm{d} P(\omega) \geq \int_{j^{-1}[1 / 2+1 / n,+\infty)}\left(\frac{1}{2}+\frac{1}{n}\right) \mathrm{d} P=\frac{1}{2}+\frac{1}{n},
$$

a contradiction. That $j^{-1}(1 / 2,+\infty)$ is countable follows, as

$$
j^{-1}(1 / 2,+\infty)=\bigcup_{n} j^{-1}[1 / 2+1 / n,+\infty) .
$$

Similarly, $j^{-1}(-\infty, 1 / 2)$ is countable. 
The proof of $E(S \mid \mathscr{A}(\tau))=1 / 2$ a.s. is to repeat the steps above, with the difference that now $j^{-1}[1 / 2+1 / n,+\infty)$ is finite.

A more subtle point is that the conditional expectation with respect to an algebra is not guaranteed to exist by the conventional theory of conditional expectation. ${ }^{5}$ In our example, the conditional expectations $E(S \mid \mathscr{A}(\tau))$ and $E\left(S \mid \mathscr{A}\left(\tau^{\prime}\right)\right)$ are well-defined.

Proof of Theorem A. $(2 \Rightarrow 1)$. If $P_{f} \geq P_{g}$, all $g$-feasible acts are $f$-feasible. The result is immediate. To prove $(1 \Rightarrow 2)$, suppose, by way of contradiction, that $P_{f}$ is not finer than $P_{g}$. Then there exist $\boldsymbol{\omega}$ and $\boldsymbol{\omega}^{\prime}$ such that $f(\boldsymbol{\omega})=f\left(\boldsymbol{\omega}^{\prime}\right)$ but $g(\boldsymbol{\omega}) \neq g\left(\boldsymbol{\omega}^{\prime}\right)$. Define, for $x \neq y$ the following act

$$
a\left(\boldsymbol{\omega}^{\prime \prime}\right)= \begin{cases}x & \text { if } g\left(\omega^{\prime \prime}\right)=g(\omega) \\ y & \text { otherwise }\end{cases}
$$

Note that $a$ is $g$-feasible but not $f$-feasible. Let $\succeq$ be a decisionmaker such that $a \succ \tilde{a}$ for all $\tilde{a} \in A$. There is no $f$-feasible $\tilde{a} \in A$ such that $\tilde{a} \succeq a$, so $\succeq$ does not prefer $f$ to $g$.

$\left(2 \Rightarrow 3\right.$ ) Suppose $P_{f} \geq P_{g}$. For each $z \in g(\Omega)$ let $Y_{f}^{z}=f\left(g^{-1}(z)\right)$ and let $\tilde{z}$ be any element in $Z$. It is easy to check that for

$$
h(y)= \begin{cases}z & \text { if } y \in Y_{f}^{z} \text { for somezin } g(\Omega) \\ \tilde{z} & \text { otherwise }\end{cases}
$$

we have $h \circ f=g$.

$\left(3 \Rightarrow 2\right.$ ) If it is not the case that $P_{f} \geq P_{g}$, there exist $\omega$ and $\omega^{\prime}$ such that $\omega$ and $\omega^{\prime}$ are in the same element of $P_{f}$ but not of $P_{g}$. Then, for all $h: Y_{f} \rightarrow Y_{g}$,

$$
h(f(\omega))=h\left(f\left(\omega^{\prime}\right)\right) \text { but } \quad g(\omega) \neq g\left(\omega^{\prime}\right)
$$

so $h \circ f \neq g$.

$(2 \Rightarrow 4)$. Notice that $Q(y, \cdot)=\delta_{g f^{-1}(y)}(\cdot)$ satisfies

$$
\begin{aligned}
T \delta_{f(\omega)}(E) & =\int Q(y, E) \mathrm{d} \delta_{f(\omega)}(y)=Q(f(\omega), E) \\
& =\delta_{g f^{-1}(f(\omega))}(E)=\delta_{g(\omega)}(E)
\end{aligned}
$$

as was sought.

$(4 \Rightarrow 2)$. By hypothesis, $\delta_{g(\omega)}(E)=\int Q(y, E) \mathrm{d} \delta_{f(\omega)}(y)$ for some $Q$. Then, $f(\omega)=f\left(\omega^{\prime}\right)$ implies that $\delta_{g(\omega)}(E)=\delta_{g\left(\omega^{\prime}\right)}(E)$. So $g(\omega)=g\left(\omega^{\prime}\right)$.

(2 $\Rightarrow 5$ ) If $P_{f} \geq P_{g}$, for every collection of sets $\left\{C_{i}\right\}$ in $P_{g}$, there exists a collection $\left\{B_{i}\right\}$ in $P_{f}$ such that $\cup_{i} C_{i}=\cup_{i} B_{i}$. Thus, letting $\alpha(\tau)$ stand for the $\sigma$-algebra of arbitrary unions of elements in $\tau, \alpha\left(P_{g}\right) \subseteq \alpha\left(P_{f}\right)$.

\footnotetext{
${ }^{5}$ The theory uses the Radon-Nykodim Theorem. There are versions of the Radon-Nykodim Theorem for measures on algebras - see Berti et al. (1992)—but we do not know that they give an existence theorem for conditional expectations with respect to an algebra.
} 
(5 $\Rightarrow 2$ ) Suppose that it is not the case that $P_{f} \geq P_{g}$. This means that there exists a set $C$ in $P_{g}$ such that for every collection $\left\{B_{i}\right\}$ in $P_{f}, C \neq \cup_{i} B_{i}$. Thus, $C \in \alpha\left(P_{g}\right)$, but $C \notin \alpha\left(P_{f}\right)$, a contradiction.

(2 $\Rightarrow 6$ ) Suppose $P_{f} \geq P_{g}$, and that $c \in \sigma(g, \mathscr{C})$. This means that there exists $C \in \mathscr{C}$ such that $c=g^{-1}(C)$. Since $P_{f} \geq P_{g}$,

$$
c=\bigcup_{i \in I} p_{i}
$$

for some index set $I$ and $p_{i} \in P_{f}$ for all $i$. Since $\mathscr{B}$ distinguishes $f, f\left(p_{i}\right) \in \mathscr{B}$ for all $i$, and since $\mathscr{B}$ is closed under arbitrary unions, $\cup_{i \in I} f\left(p_{i}\right) \in \mathscr{B}$. We then obtain that

$$
c=f^{-1}\left(\bigcup_{i \in I} f\left(p_{i}\right)\right) \in \sigma(f, \mathscr{B}) .
$$

$(6 \Rightarrow 2)$ It is easy to check that since $\mathscr{B}$ ( $\mathscr{C}$ resp.) distinguishes $f(g$ resp. $), \sigma(f, \mathscr{B})$ $(\sigma(g, \mathscr{C})$ resp. $)$ is the collection of arbitrary unions of elements of $P_{f}$ ( $P_{g}$ resp.). Therefore, for all $c \in P_{g}$ we have $c \in \sigma(g, \mathscr{C})$, and by hypothesis, $c \in \sigma(f, \mathscr{B})$. But then, there must exist a collection $\left\{p_{i}\right\}_{i \in I}$ such that $p_{i} \in P_{f}$ for all $i$, and $c=\cup_{i \in I} p_{i}$, so $P_{f} \geq P_{g}$.

\section{References}

Berti, P., Regazzini, E., Rigo, P., 1992. Finitely additive Radon-Nikodym theorem and concentration function of a probability with respect to a probability. Proceedings of the American Mathematical Society 114 (4), $1069-1078$

Billingsley, P., 1995. Probability and Measure. J. Wiley.

Blackwell, D., 1951. Comparison of experiments. In: Neyman, J. (Ed.), Proceedings of the Second Berkeley Symposium on Mathematical Statistics and Probability, University of California Press, Berkeley, pp. 93 - 102.

Blackwell, D., 1953. Equivalent comparisons of experiments. Annals of Mathematical Statistics 24 (2), 93 - 102.

Blackwell, D., 1956. On a class of probability spaces. In: Neyman, J. (Ed.), Proceedings of the Third Berkeley Symposium on Mathematical Statistics and Probability, University of California Press, Berkeley, pp. 1-6.

Brandenburger, A., Dekel, E., 1993. Hierarchies of beliefs and common knowledge. Journal of Economic Theory 59 (1), 189-198.

Dudley, R.M., 1989. Real Analysis and Probability. Chapman and Hall.

Mertens, J.-F., Zamir, S., 1985. Formulation of Bayesian analysis for games of incomplete information. International Journal of Game Theory 14, 1-29.

Stinchcombe, M.B., 1990. Bayesian information topologies. Journal of Mathematical Economics 19, 233 - 253. Yannelis, N.C., 1991. The core of an economy with differential information. Economic Theory 1 (2), $183-197$. 